\title{
Protective effects of human induced pluripotent stem cell-derived exosomes on high glucose-induced injury in human endothelial cells
}

\author{
QIANQIAN DING, RUITING SUN, PINGPING WANG, HENG ZHANG, MENG XIANG, \\ DAN MENG, NING SUN, ALEX F. CHEN and SIFENG CHEN
}

Department of Pathophysiology, School of Basic Medical Sciences, Fudan University, Shanghai 200032, P.R. China

Received July 14, 2016; Accepted August 25, 2017

DOI: $10.3892 /$ etm.2018.6059

\begin{abstract}
Exosomes are a family of extracellular vesicles that are secreted from almost all types of cells and are associated with cell-to-cell communication. The present study was performed to investigate the effects of human induced pluripotent stem cell-derived exosomes (hiPSC-exo) on cell viability, capillary-like structure formation and senescence in endothelial cells exposed to high glucose. Exosomes were isolated from the conditional medium of hiPSCs and confirmed by transmission electron microscopy, nanoparticle tracking analysis and western blot analysis using Alix and cluster of differentiation-63 as markers. hiPSC-exo were labeled with PKH26 for tracking, and it was determined that spherical exosomes, with a typical cup-shape, were absorbed by human umbilical vascular endothelial cells (HUVECs). Cultured HUVECs were treated with high glucose $(33 \mathrm{mM})$ with or without hiPSC-exo $(20 \mu \mathrm{g} / \mathrm{ml})$ for $48 \mathrm{~h}$, and cell viability, capillary tube formation and senescence were assessed. When exposed to high glucose, viability and tube formation in HUVECs was significantly reduced $(\mathrm{P}<0.0001)$, whereas the proportion of senescent cells was higher compared with that in control HUVECs $(\mathrm{P}<0.0001)$. Furthermore, hiPSC-exo restored cell viability and capillary-like structure formation, and reduced senescence in HUVECs exposed to high glucose $(\mathrm{P}<0.0001)$. However, hiPSC-exo had minimal effects on normal HUVECs. These findings suggest that stem
\end{abstract}

Correspondence to: Professor Sifeng Chen, Department of Pathophysiology, School of Basic Medical Sciences, Fudan University, Room 214, Building 7, 130 Dongan Road, Shanghai 200032, P.R. China

E-mail: chen1216@fudan.edu.cn

Abbreviations: hiPSC-exo, human induced pluripotent stem cell-derived exosomes; HUVECs, human umbilical vascular endothelial cells

Key words: induced pluripotent stem cells, exosomes, endothelial cells, high glucose, repair cell-derived exosomes are able to promote cell proliferation, enhance capillary-like structure formation and reduce senescence in endothelial cells exposed to high glucose.

\section{Introduction}

Patients with type 2 diabetes mellitus are at a notably high risk for developing life-threatening cardiovascular complications (1), and there is a clear association between glycemic control and cardiovascular diseases (2). Unlike vascular smooth muscle cells, endothelial cells cannot regulate intrinsic glucose levels, which can result in the accumulation of glucose and its derivatives, leading to an array of metabolic disorders. Furthermore, glucose toxicity can cause reduced cell viability and increased senescence in endothelial cells via multiple signaling pathways. The cardiovascular complications of diabetes have a close association with the function of endothelial cells; however, the underlying mechanism needs further investigation (3-5). Therefore, endothelial cells are potential targets for preventing the cardiovascular complications of diabetes.

Induced pluripotent stem cells (iPSCs) may be used to treat a number of diseases, as they possess the potential for self-renewal and multi-differentiation (6). It has previously been suggested that transplanted iPSCs were able to inhibit vascular apoptosis and fibrosis, thus improving cardiac function in diabetic rats (7). However, little is known about the mechanisms by which iPSCs, or factors released from these cells, inhibit adverse cardiac remodeling (8). Despite their impressive therapeutic ability, teratoma formation has been observed following transplantation of iPSCs $(9,10)$. iPSC-derived lineage cells have avoided this issue; however, the derivatives may still suffer the same difficulties as reported for adult stem cells, particularly in cell survival, retention and coupling in damaged areas (11). Therefore, it is important to exploit the powerful regenerative capacity of pluripotent stem cells while circumventing the problems associated with cell transplantation.

The discovery of cell-free components, including exosomes, may provide a promising alternative for regenerative medicine. Exosomes are small membrane vesicles that contain membrane and cytosolic components, including proteins, 
lipids and RNAs (12-14). They have an essential role in intercellular communication via transporting this cargo to targeted cells. Furthermore, the role of exosomes depends on their components and cell origin. It has been reported that adipose-derived mesenchymal stromal cells release exosomes that are capable of promoting angiogenesis (15). In addition, embryonic stem cell-derived exosomes have been shown to enhance cell proliferation (16). As one of the most dynamic cells with regenerative potential, iPSCs may also release exosomes, suggesting further potential for disease treatment.

In the present study, human induced pluripotent stem cell-derived exosomes (hiPSC-exo) were isolated and were co-cultured with normal human umbilical vascular endothelial cells (HUVECs) or HUVECs exposed to high glucose. Subsequently, the cell viability, capacity to form capillary-like structures and cell senescence was examined to demonstrate the role for hiPSC-exo in endothelial cell growth.

\section{Materials and methods}

Cell culture. HiPSCs were induced at the Cardiovascular Regenerative Engineering Laboratory (Shanghai, China), following a previously described protocol (17) and cultured in mTeSR (Stemcell Technologies, Inc., Vancouver, Canada), which contains mTeSR1 basal medium (400 ml; cat. no. 05851) and mTeSR1 5X supplement (100 ml; cat. no. 05852). The culture medium was replaced every day, and the cells were digested with Accutase (cat. no. A6964; Sigma-Aldrich; Merck KGaA, Darmstadt, Germany) and passaged at a 1:5 dilution every 3 days. Following the culture of passaged cells for $48 \mathrm{~h}$ at $37^{\circ} \mathrm{C}$ in a $5 \% \mathrm{CO}_{2}$ and $95 \%$ air gas mixture atmosphere, the media was changed. The media was then collected for exosome isolation following a further incubation with cells at $37^{\circ} \mathrm{C}$ in a $5 \% \mathrm{CO}_{2}$ and $95 \%$ air gas mixture atmosphere for $24 \mathrm{~h}$.

Primary HUVECs were isolateded from fresh umbilical cord veins from 3 patients that had undergone normal pregnancy, $24 \mathrm{~h}$ following birth, from June 2015 to December of 2015 at the Department of Obstetrics, The Obstetrics and Gynecology Hospital of Fudan University (Shanghai, China). For the collection of HUVECS, informed consent was obtained from all patients and ethical approval was granted by the Experimental Animal and Ethics Committee of the College of Basic Medical Sciences at Fudan University (Shanghai, China). HUVECs were isolated by collagenase digestion as previously described (18) and cultured in endothelial cell medium (ScienCell Research Laboratories, Inc., San Diego, CA, USA) supplemented with 5\% fetal bovine serum (cat. no. 0025), 1\% endothelial cell growth supplement (cat. no. 1052) and $1 \%$ penicillin/streptomycin solution (cat. no. 0503). Furthermore, the culture medium was replaced every 2 days. When the HUVECs became $80 \%$ confluent, cells were sub-cultured with $0.25 \%$ trypsin-EDTA and phenol red 1X (cat. no. 25200072; Thermo Fisher Scientific Inc., Waltham, MA, USA), and were incubated at $37^{\circ} \mathrm{C}$ in an atmosphere containing $5 \% \mathrm{CO}_{2}$.

Exosome isolation. Exosomes from hiPSC were collected and purified using ultracentrifugation. Briefly, timing began when hiPSCs were $70 \%$ confluent. After 48 h, $80 \mathrm{ml}$ cell-conditioned medium was collected and centrifuged at 3,000 x g for $30 \mathrm{~min}$ at $4^{\circ} \mathrm{C}$ and $10,000 \times \mathrm{g}$ for $30 \mathrm{~min}$ at $4^{\circ} \mathrm{C}$ to remove dead cells and cell debris. The final supernatant was ultracentrifuged and exosomes were pelleted at $100,000 \mathrm{x}$ g for $70 \mathrm{~min}$ at $4^{\circ} \mathrm{C}$. The pellet obtained was then washed with PBS at 100,000 x g for $70 \mathrm{~min}$ at $4^{\circ} \mathrm{C}$ to eliminate contaminating proteins. The purified exosome fraction was re-suspended in PBS prior to further use.

HiPSC-exo labeling and uptake assay. Exosomes were labeled using a PKH26 Red Fluorescent Cell Linker kit (PKH26GL-1KT; Sigma-Aldrich; Merck KGaA) according to the manufacturer's protocol. A total of $1 \mu \mathrm{l} \mathrm{PKH} 26$ was mixed with $250 \mu 1$ Diluent $C$ (provided by the kit). HiPSC-exo with the same volume was then added into the mixture and incubated at room temperature for $5 \mathrm{~min}$. Labeling was stopped by adding $500 \mu 10.5 \%$ bovine serum albumin followed by an incubation at room temperature for $5 \mathrm{~min}$. The exosomes were then centrifuged at $100,000 \times \mathrm{g}$ for $70 \mathrm{~min}$ at $4^{\circ} \mathrm{C}$ and re-suspended in PBS prior to the uptake assay. For the uptake assay, HUVECs were seeded in 96-well plates and left to proliferate for $24 \mathrm{~h}$ at $37^{\circ} \mathrm{C}$ in a $5 \% \mathrm{CO}_{2}$ and $95 \%$ air gas mixture. Following $24 \mathrm{~h}$, 20, $50 \mu \mathrm{g} / \mathrm{ml}$ hiPSC-exo or the PBS control were added into the dish and cultured for a further $24 \mathrm{~h}$ at $37^{\circ} \mathrm{C}$ in a $5 \% \mathrm{CO}_{2}$ and $95 \%$ air gas mixture. The following steps were applied to the cells prior to observation under an inverted phase contrast microscope at a magnification of x 20 . The cells were washed with PBS twice, fixed with freshly prepared $4 \%$ paraformaldehyde in PBS at room temperature for $15 \mathrm{~min}$, further washed twice with PBS and incubated with $0.1 \%$ triton X-100/PBS at room temperature for $15 \mathrm{~min}$. Cells were subsequently washed twice with PBS, stained with DAPI (1:5,000; cat. no. C1002; Beyotime, Shanghai, China) for $5 \mathrm{~min}$ at room temperature and further washed with PBS.

Transmission electron microscopy. Transmission electron microscopy images of hiPSC-exo were obtained using an FEI Tecnai G2 Spirit twin transmission electron microscope (FEI; Thermo Fisher Scientific, Inc.) operating at $80 \mathrm{kV}$. Briefly, $10 \mu 1$ hiPSC-exo sample was loaded onto a formvar-carbon coated copper grid for 2 min and excess sample was absorbed with filter paper by gently touching the edge of the grid and removed. Next, uranyl acetate was used to stain the grid for $1 \mathrm{~min}$ at room temperature and the excess liquid was absorbed using filter paper. The copper grid was then dried under an incandescent lamp.

Nanoparticle tracking analysis. Exosome size and concentration analysis was performed using Nanoparticle Tracking Analyzer (version, 3.1; Build, 3.1.54; Malvern Instruments, Ltd., Worcestershire, UK). For the measurement, $10 \mu \mathrm{l}$ hiPSC-exo sample was diluted in $1 \mathrm{ml}$ of PBS.

Cell counting assay. A cell counting kit-8 (CCK-8; cat. no. CK04; Dojindo Molecular Technologies, Inc., Kumamoto, Japan) was used to identify the effect of hiPSC-exo on the viability of HUVECs. For this assay, the same initial number $\left(1 \times 10^{3}\right)$ of HUVECs was seeded in 96-well plates and allowed to proliferate for $24 \mathrm{~h}$ at $37^{\circ} \mathrm{C}$, in a $5 \% \mathrm{CO}_{2}$ and $95 \%$ air gas mixture. The cells were then treated with different conditions [normal glucose (5 mM); normal glucose + hiPSC-exo $(10,20$ or $50 \mu \mathrm{g} / \mathrm{ml})$; 
high glucose $(33 \mathrm{mM})$ and high glucose + hiPSC-exo (10, 20 or $50 \mu \mathrm{g} / \mathrm{ml})$ for a further $48 \mathrm{~h}$ at $37^{\circ} \mathrm{C}$ in a $5 \% \mathrm{CO}_{2}$ and 95\% air gas mixture. The culture media were aspirated and CCK-8 mixed media (CCK-8: Dulbecco's modified Eagle's medium, 1:10) was added to the different groups. After $2 \mathrm{~h}$, the absorbance was measured at $450 \mathrm{~nm}$ using a spectrophotometer microplate reader. The assays were performed in sextuplicate and each experiment was repeated five times.

Capillary-like structure formation assay. A capillary-like structure formation assay was performed to identify the functional role of hiPSC-exo on endothelial cells. Briefly, HUVECs $\left(1 \times 10^{4}\right.$ cells) were cultured in 24 -well plates at $37^{\circ} \mathrm{C}$ in a $5 \% \mathrm{CO}_{2}$ and $95 \%$ air gas mixture for $24 \mathrm{~h}$ and subjected to different treatments (normal glucose $(5 \mathrm{mM})$, normal glucose + hiPSC-exo $(20 \mu \mathrm{g} / \mathrm{ml})$, high glucose $(33 \mathrm{mM})$ and high glucose + hiPSC-exo $(20 \mu \mathrm{g} / \mathrm{ml})$ for another $48 \mathrm{~h}$ at $37^{\circ} \mathrm{C}$, in a $5 \% \mathrm{CO}_{2}$ and $95 \%$ air gas mixture. After $48 \mathrm{~h}$, HUVECs were trypsinized and cultured in 24-well plates $\left(1 \times 10^{5}\right.$ cells/well) coated with $200 \mu 1$ Matrigel matrix (cat. no. 356234; BD Biosciences, Franklin Lakes, NJ, USA). Tube length was quantified after $6 \mathrm{~h}$ by calculating the mean length in five random microscopic fields with an inverted phase contrast microscope at magnification, x10. ImageJ software (version, 1.42q) was then used to analyze the data (National Institutes of Health, Bethesda, MD, USA). Each experiment was repeated three times.

Senescence-associated staining. Senescence-associated staining was applied to demonstrate that hiPSC-exo could inhibit cell senescence in HUVECs. Briefly, HUVECs $\left(1 \times 10^{4}\right.$ cells) were cultured in $24-w e l l$ plates at $37^{\circ} \mathrm{C}$ with $5 \% \mathrm{CO}_{2}$ for $24 \mathrm{~h}$ and treated with normal glucose $(5 \mathrm{mM})$, normal glucose + hiPSC-exo $(20 \mu \mathrm{g} / \mathrm{ml})$, high glucose $(33 \mathrm{mM})$ and high glucose + hiPSC-exo $(20 \mu \mathrm{g} / \mathrm{ml})$ for a further $48 \mathrm{~h}$ at $37^{\circ} \mathrm{C}$, in a $5 \% \mathrm{CO}_{2}$ and $95 \%$ air gas mixture. Subsequently, senescence-associated $\beta$-galactosidase (SA- $\beta$-gal) staining was performed using an SA- $\beta$-gal staining kit (cat. no. K320-250; BioVision, Inc., Milpitas, CA, USA) according to the manufacturer's instructions. HUVECs were washed three times with PBS and fixed for $15 \mathrm{~min}$ at room temperature with fixative solution. After incubation with staining solution overnight at $37^{\circ} \mathrm{C}$, senescence was quantified by calculating mean proportion of senescent cells in five random microscopic fields using an inverted phase contrast microscope at magnification, x20 and ImageJ software. Each experiment was repeated three times.

Western blot analysis. hiPSCs and hiPSC-exo were lysed using radioimmunoprecipitation assay buffer (cat. no. 89901; Thermo Fisher Scientific, Inc.) for $30 \mathrm{~min}$ on ice, followed by a centrifugation at a speed of $12,000 \mathrm{x}$ g for $20 \mathrm{~min}$ at $4^{\circ} \mathrm{C}$. The concentration of the protein was determined via bicinchoninic acid assay (Pierce BCA Protein Assay Reagent A; cat. no. 23227; Thermo Fisher Scientific, Inc.). A total of $20 \mu \mathrm{g}$ protein was mixed with 5X SDS loading buffer and loaded onto 10\% SDS-PAGE. The separated protein bands in the gel were transferred onto a polyvinylidene difluoride (PVDF) membrane. The membrane was then blocked with 5\% non-fat milk in PBS with 0.1\% Tween-20 (PBST) at room temperature for $2 \mathrm{~h}$. PVDF membranes were then incubated with primary antibodies at $4^{\circ} \mathrm{C}$ overnight and then washed with PBST. Secondary antibodies were added to each blot, and incubated at room temperature for $2 \mathrm{~h}$; PVDF membranes were then washed with PBST and incubated with Super Signal ${ }^{\mathrm{TM}}$ West Pico (cat. no. 34580; Thermo Fisher Scientific, Inc.) and observed using a Western blot visualizer (Tanon 5500; Tanon Science \& Technology Co., Ltd., Shanghai, China). Primary antibodies used for exosome identification were goat anti-Alix (N-20; 1:1,000; cat. no. sc-49267; Santa Cruz Biotechnology Inc., Dallas, TX) and anti-cluster of differentiation (CD63; rabbit IgG; 1:1,000; cat. no. EXOAB-CD63A-1; System Biosciences Inc., Palo Alto, CA). Secondary antibodies used were horseradish peroxidase (HRP)-conjugated donkey anti-goat $\operatorname{IgG}(1: 2,000$; cat. no. 705-035-003; Jackson ImmunoResearch Laboratories Inc., West Grove, PA) and HRP-conjugated donkey anti-rabbit IgG (1:2,000; cat. no. 711-005-152; Jackson ImmunoResearch Laboratories Inc.). The experiment was repeated three times.

Statistical analysis. Statistical analysis was performed using one-way analysis of variance and a post-hoc Bonferroni test. $\mathrm{P}<0.05$ was considered to indicate a statistically significant difference. Data are presented as the mean \pm standard error of the mean, unless otherwise stated. Furthermore, statistical analysis was performed using GraphPad Prism 6.07 (GraphPad Software, Inc., La Jolla, CA) and SPSS Statistics 17.0 (SPSS Inc., Chicago, IL, USA).

\section{Results}

Identification of exosomes derived from hiPSC. A transmission electron microscopic observation of hiPSC-exo showed the presence of spherical vesicles with a typical cup-shape (Fig. 1A). Upon conducting nanoparticle tracking analysis, the concentration/size graph and particle pictorial diagram revealed a homogeneous population of exosomes ranging between 50 and $150 \mathrm{~nm}$ (Fig. 1B and C). The mode size of hiPSC-exo was $103.1 \mathrm{~nm}$, and the total concentration of particles with a diameter between 30 and $150 \mathrm{~nm}$ was $6 \times 10^{10}$ particles $/ \mathrm{ml}$ (Fig. 1D). Additionally, hiPSC-exo expressed the exosomal marker proteins Alix and CD63 (Fig. 1E).

Uptake of hiPSC-exo by HUVECs. In order to function, hiPSC-exo requires the ability to be endocytosed by target cells. Previous research has demonstrated that exosomes express adhesion molecules that may be associated with the adherence of exosomes to cells; however, the cellular and molecular basis for specific targeting to acceptor cells remains to be elucidated. For example, exosomes released by the human intestinal epithelial cell line T84 could be endocytosed by dendritic cells (DCs), but not B or T lymphocytes (19). The present study therefore tested if hiPSC-exo could be taken up by HUVECs. It was demonstrated in Fig. 2A and B that when HUVECs were treated with PKH26 labeled hiPSC-exo (20 and $50 \mu \mathrm{g} / \mathrm{ml}$ ) exosomes were endocytosed by cells in a concentration-dependent manner. By contrast, the control group, which was subjected to the same procedure, did not show any intracellular fluorescence as shown in Fig. 2C.

hiPSC-exo reversed high glucose-induced decreased cell viability. Fig. 2 demonstrated that hiPSC-exo may be endocytosed 
A

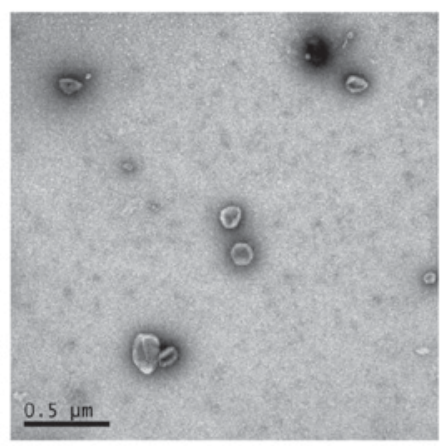

C

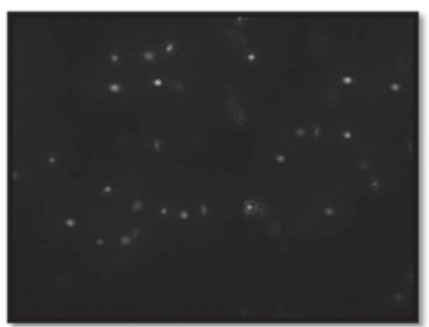

B

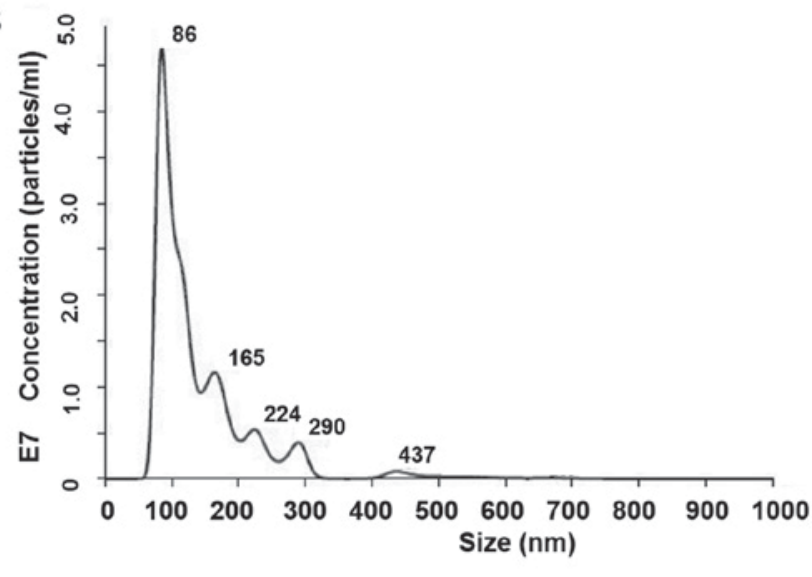

$\mathbf{E}$

\begin{tabular}{|l|l|}
\hline Mode & $103.1 \mathrm{~nm}$ \\
\hline $\mathrm{SD}$ & $58.4 \mathrm{~nm}$ \\
\hline Concentration & $6 \mathrm{E} 10$ particle $/ \mathrm{ml}$ \\
\hline
\end{tabular}

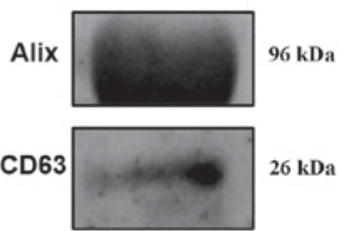

Figure 1. Characterization of hiPSC-exo. (A) Transmission electron microscopy of hiPSC-exo. Scale bar, $0.5 \mu \mathrm{m}$. (B-D) NTA of hiPSC-exo fraction. (B) A graph plotting the concentration vs. particle size was obtained from exosome measurements. (C) A particle pictorial diagram. (D) Mode particle size of hiPSC-exo obtained by NTA measurements. (E) Alix and CD63 expression in hiPSC-exo detected by western blot analysis. hiPSC-exo, human induced pluripotent stem cell-derived exosomes; NTA, Nanoparticle tracking analysis; SD, standard deviation; CD, cluster of differentiation.

by HUVECs. It was then investigated if endocytosed hiPSC-exo could influence the fate of HUVECs. In CCK- 8 assay the amount of formazan dye in cells is directly proportional to the number of living cells. In the present study, HUVECs were treated with normal $(5.5 \mathrm{mM})$ or high $(33 \mathrm{mM})$ concentrations of glucose, in combination with different concentrations $(0,10,20$ or $50 \mu \mathrm{g} / \mathrm{ml}$ ) of hiPSC-exo. After $48 \mathrm{~h}$ treatment, the OD value of the eight groups was measured. Compared with the control group [normal concentration of glucose $(5.5 \mathrm{mM})]$, the OD value for high glucose $(33 \mathrm{mM})$ treated HUVECs was significantly decreased (Fig. 3). Furthermore, exosomes had no statistically significant effect on normal HUVECs. HiPSC-exo significantly reversed the harmful effect of high glucose. However, the effects between hiPSC-exo 20 and $50 \mu \mathrm{g} / \mathrm{ml}$ did not differ significantly (Fig. 3). Thus, a concentration of $20 \mu \mathrm{g} / \mathrm{ml}$ was selected for use in subsequent experiments.

hiPSC-exo reversed high glucose-induced decreases in capillary-like structure formation. Following treatment with normal glucose, normal glucose + hiPSC-exo, high glucose or high glucose + hiPSC-exo, HUVECs were trypsinized and cultured on Matrigel to assess in vitro capillary-like structure formation. The results demonstrated that high glucose significantly decreased capillary-like structure formation in HUVECs, whereas hiPSC-exo reversed this effect. However, hiPSC-exo had a minimal effects on normal HUVECs (Fig. 4).

Anti-senescence effect of hiPSC-exo in high glucose-injured HUVECs. To verify the effects of hiPSC-exo on cell senescence, a senescence detection assay was performed on
HUVECs after being treated with different conditions (normal glucose, normal glucose + hiPSC-exo, high glucose and high glucose + hiPSC-exo). The cells of the control group were transparent and plump whereas the cells of the high glucose group showed a flattened and enlarged morphology (Fig. 5). hiPSC-exo significantly protected HUVECs against cellular senescence induced by high glucose (Fig. 5). Furthermore, there was no statistically significant difference between the normal glucose group and either group treated with hiPSC-exo. Collectively, the results showed that hiPSC-exo are readily absorbed by target cells, wherein they can modulate cell viability and survival.

\section{Discussion}

To the best of our knowledge, this is the first report demonstrating that exosomes derived from hiPSCs are able to protect HUVECs from high glucose in vitro. In the present study, HUVECs were found to be capable of absorbing hiPSC-exo at a high efficiency. The mechanism of exosome uptake by targeted cells has long been studied. The existing model suggests that exosomes express adhesion molecules to adhere to cells (20); however, the cellular and molecular basis for their specific targeting to acceptor cells remains to be elucidated. Barrès et al (21) demonstrated previously that galectin-5 was bound to the surface of rat reticulocyte exosomes and modulated vesicle uptake by macrophages. In the immune system, it has been demonstrated that $\mathrm{T}$ cells were able to recruit major histocompatibility complex class II-containing DC exosomes, and that this recruitment was dependent on leukocyte function-associated antigen-1 (22). 

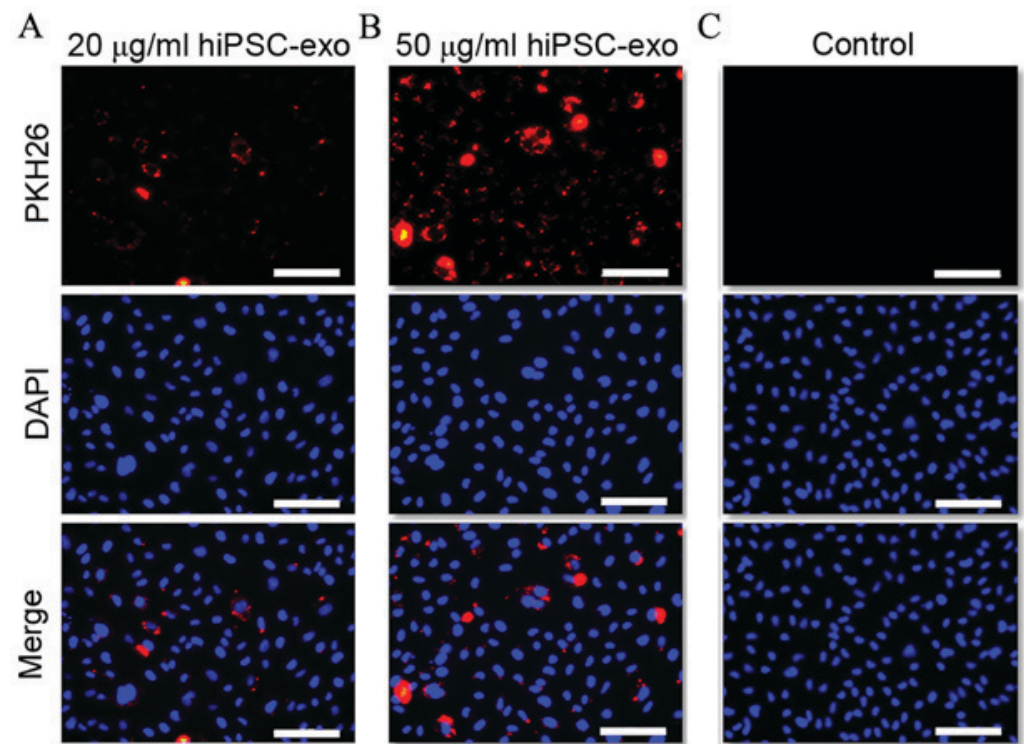

Figure 2. Uptake of hiPSC-exo by HUVECs. Inverted fluorescence microscopy confirmed the location of hiPSC-exo in HUVECs following $24 \mathrm{~h}$ treatment with (A) 20 or (B) $50 \mu \mathrm{g} / \mathrm{ml}$ hiPSC-exo. (C) The same methods were used to show the absence of hiPSC-exo or nonspecific localization of labeling dye (control conditions). Red, hiPSC-exo labeled by PKH26; blue, nucleus of HUVECs. Scale bar, $100 \mu \mathrm{m}$. hiPSC-exo, human induced pluripotent stem cell-derived exosomes; HUVECs, human umbilical vascular endothelial cells; DAPI, 4', 6-diamidine-2'-phenylindole dihydrochloride.

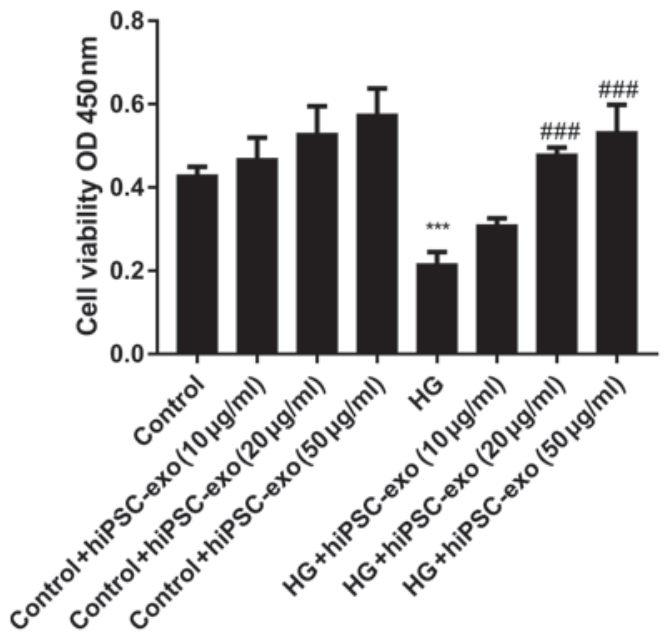

Figure 3. hiPSC-exo ameliorated the high glucose-induced decrease in cell viability in a dose-dependent manner. Cell counting kit-8 assays were performed to evaluate cell viability. HUVECs $\left(1 \times 10^{3}\right)$ were seeded in 96-well plates. The cell viability was assessed by measuring the OD value of HUVECs after $48 \mathrm{~h}$ of treatment. Control, normal glucose ( $5.5 \mathrm{mM})$; HG, high glucose (33 mM); hiPSC-exo (10, 20 or $50 \mu \mathrm{g} / \mathrm{ml})$. Control vs. HG, ${ }^{* * * *} \mathrm{P}<0.0001, \mathrm{n}=5$; HG vs. HG + hiPSC-exo $(20 \mu \mathrm{g} / \mathrm{ml}),{ }^{\# \# \#} \mathrm{P}<0.0001, \mathrm{n}=5$; HG vs. HG + hiPSC-exo $(50 \mu \mathrm{g} / \mathrm{ml}),{ }^{\# \# \#} \mathrm{P}<0.0001, \mathrm{n}=5$. hiPSC-exo, human induced pluripotent stem cell-derived exosomes; HUVECs, human umbilical vascular endothelial cells; OD, optical density; HG, high glucose.

The function of exosomes in physiological and pathological conditions depends on their cellular origin and contents. As one of the most dynamic types of cell, hiPSCs have the capacity of self-renewal and multi-differentiation, thus they exert a therapeutic effect when used to treat various diseases, including myocardial infarction $(23,24)$. The role of exosomes derived from hiPSCs was explored to examine their therapeutic effects. The results of the present study demonstrated that hiPSC-exo could promote cell viability and enhance tube formation, and inhibit cell senescence in HUVECs injured by high glucose. A number of previous studies have demonstrated that exosomes from different types of cells exert different effects. Bang et al (25) revealed that cardiac fibroblasts secreted exosomes to mediate cardiomyocyte hypertrophy, suggesting that this is a potential therapeutic target.Intravenous administration of cell-free mesenchymal stromal cells (MSCs)-generated exosomes improved functional recovery and enhanced neurite remodeling, neurogenesis and angiogenesis following stroke in rats (26), suggesting that exosomes may be important in cell therapy. A previous study by Li et al (27) demonstrated that exosomes derived from liver nonparenchymal cells mediated the cell-to-cell transmission of interferon type I- $\alpha$-induced antiviral activity. However, not all types of exosomes mediate cell-protective effects on target cells. Currently, the role of exosomes in tumors is being accepted, particularly with regard to tumor metastasis. The observations of the recent study by Zhang et al (28), demonstrated the dynamic and reciprocal cross-talk between tumor cells and the metastatic niche. They found that exosomes prepared the microenvironment of the target organ of metastasis for tumor cell colonization. Another study also demonstrated that tumor exosome integrins determine organotropic metastasis. It also revealed that exosomes from mouse and human lung-, liver- and brain-tropic tumor cells preferentially fuse with resident cells at the predicted destination (29).

In recent years, the incidence of diabetes mellitus has increased significantly, and its chronic vascular complications continue to affect patients' lives (29-31). Although the underlying specific mechanism is unclear, a growing body of research has indicated that the intracellular overproduction of reactive oxygen species (ROS), caused by hyperglycemia, is the common mechanism of vascular complications of diabetes mellitus. In a previous study by Kiritoshi et al (30), it was suggested that hyperglycemia increases mitochondrial ROS production, resulting in nuclear factor- $\kappa \mathrm{B}$ activation, cyclooxygenase (COX)-2 mRNA induction, $\mathrm{COX}-2$ protein production 

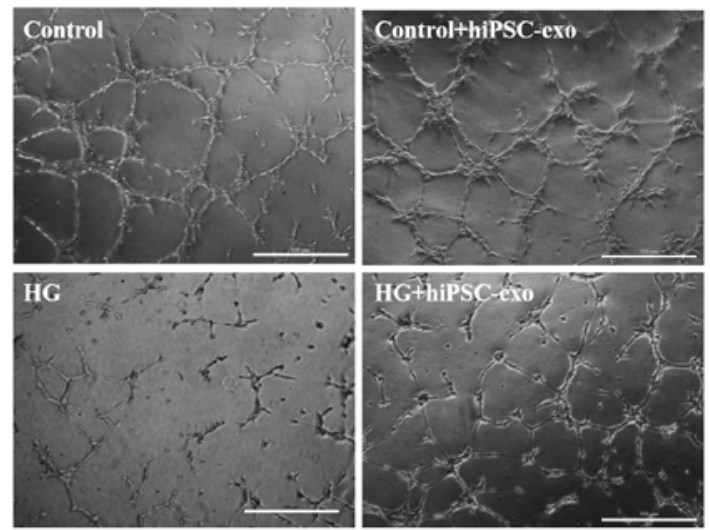

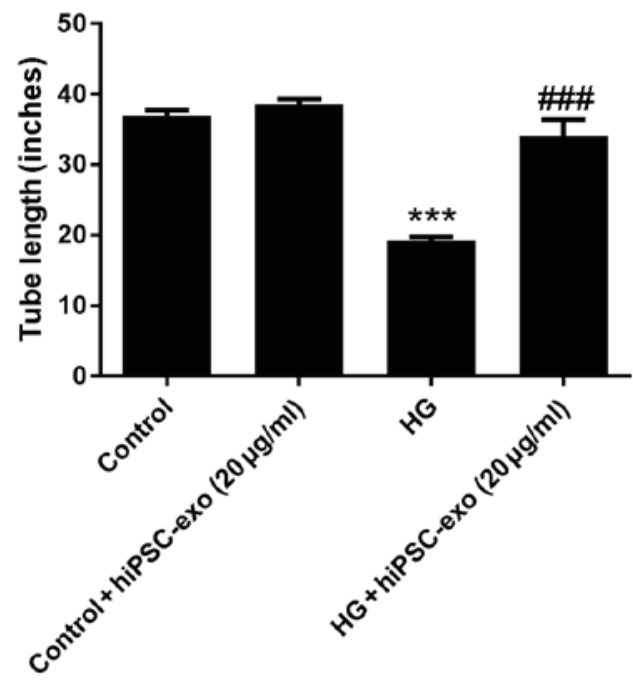

Figure 4. Enhanced capillary-like structure formation in HUVECs treated with hiPSC-exo in the presence of HG. Endothelial capillary-like structure formation was evaluated in HUVECs after being treated for $48 \mathrm{~h}$ with different conditions. Control: normal glucose (5.5 mM); HG: high glucose (33 mM); hiPSC-exo: $20 \mu \mathrm{g} / \mathrm{ml}$. The data were analyzed using one-way analysis of variance and a post-hoc Bonferroni test. Error bars represent the standard error of the mean. Control vs. HG, ${ }^{* * *} \mathrm{P}<0.0001, \mathrm{n}=3$; HG vs. HG + hiPSC-exo, ${ }^{\# \# \#} \mathrm{P}<0.0001, \mathrm{n}=3$. Scale bar, $500 \mu \mathrm{m}$. HUVECs, human umbilical vascular endothelial cells; hiPSC-exo, human induced pluripotent stem cell-derived exosomes; HG, high glucose.
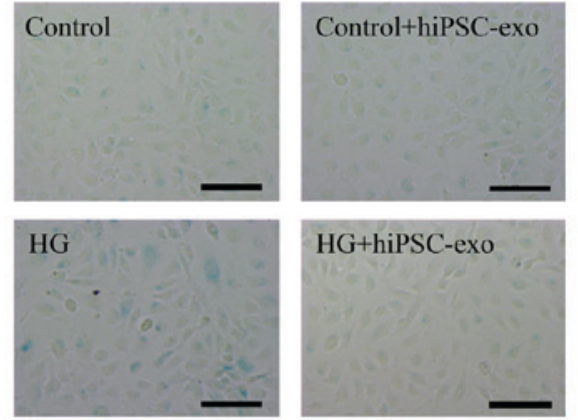

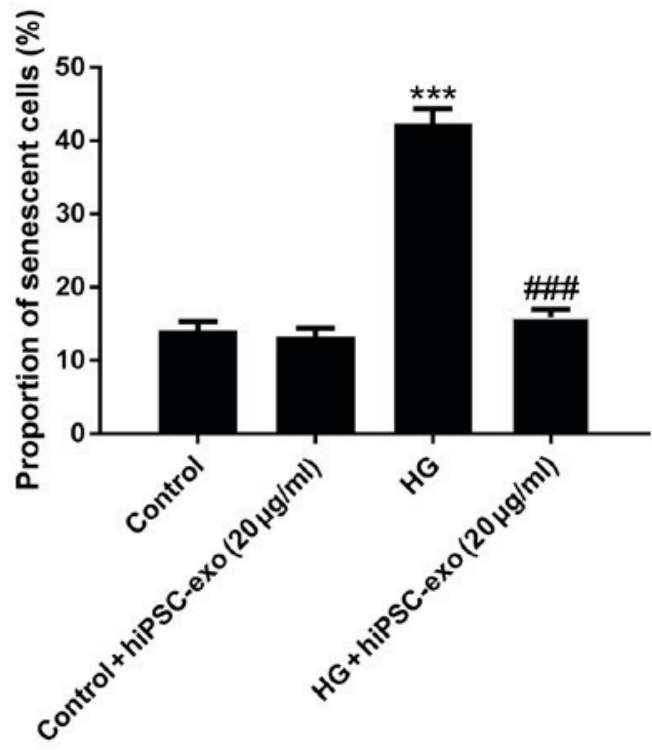

Figure 5. Effects of hiPSC-exo on the proportion of SA- $\beta$-gal positive HUVECs with different treatments. Normal glucose (5.5 mM); normal glucose + hiPSC-exo $(20 \mu \mathrm{g} / \mathrm{ml}) ; \mathrm{HG}(33 \mathrm{mM}) ; \mathrm{HG}+$ hiPSC-exo $(20 \mu \mathrm{g} / \mathrm{ml})$. Representative images of SA- $\beta$-gal positive cells (green) are presented in the left panel. Scale bar, $100 \mu \mathrm{m}$. Quantification of senescence assay is presented in the right panel. The data were analyzed using one-way analysis of variance and a post-hoc Bonferroni test. Error bars represent the standard error of the mean. Control vs. HG, ${ }^{* * *} \mathrm{P}<0.0001, \mathrm{n}=3$; HG vs. HG + hiPSC-exo, ${ }^{\# \#} \mathrm{P}<0.0001, \mathrm{n}=3$. hiPSC-exo, human induced pluripotent stem cell-derived exosomes; SA- $\beta$-gal, senescence-associated $\beta$-galactosidase; HUVECs, human umbilical vascular endothelial cells; HG, high glucose.

and prostaglandin E2 synthesis. Yu et al (31) previously determined that a dynamic change in mitochondrial morphology in high glucose conditions contributed to the overproduction of ROS. The present study indicated that mitochondrial fission/fusion machinery may be a previously unrecognized target to control acute and chronic production of ROS in hyperglycemia-associated disorders. Therefore, it is vital to understand the specific mechanisms of ROS overproduction and resulting vascular complications of diabetes mellitus, and to find novel strategies and drugs to treat this problem. This is necessary to prevent or reduce the dysfunction of endothelial cells, which are important in maintaining vascular function. A number of studies have demonstrated the therapeutic effects of exosomes from different types of cells on normal and injured endothelial cells. Conigliaro et al (32) found that exosomes released by CD90+ cancer cells modulated endothelial cells, promoting an angiogenic phenotype and cell-to-cell adhesion. When rats with traumatic brain injury were treated with MSC-derived exosomes, Zhang et al (33) found that newly generated endothelial cells in the lesion boundary zone were significantly increased with a parallel reduction in neuroinflammation. 
Therefore, the present study may offer a novel strategy for maintaining the normal function of endothelial cells during the vascular complications of diabetes mellitus.

\section{Acknowledgements}

The abstract was presented at a meeting of the 13th Congress of the International Society of Heart Research (ISHR) Chinese Section, which took place on September 21-25, 2016, Wuhan, China. The present study was supported by International Cooperation and Exchanges (81220108002 to S. Chen), Great Research Plan Program (91539120 to S. Chen), and General Program (81470260 to M. Xiang) of the National Natural Science Foundation of China, and the National Key R\&D Program of China (2016YFC1305101 to S. Chen).

\section{References}

1. Boyle PJ: Diabetes mellitus and macrovascular disease Mechanisms and mediators. Am J Med 120 (9 Suppl 2): S12-S17, 2007.

2. Sun KX, Liu ZK, Cao YY, Juan J, Xiang X, Yang C, Huang SP, Liu XF, Li N, Tang X, et al: Relationship between brachial-ankle pulse wave velocity and glycemic control of type 2 diabetes mellitus patients in Beijing community population. Beijing Da Xue Xue Bao Yi Xue Ban 47: 431-436, 2015 (In Chinese).

3. Hermans MP: Diabetes and the endothelium. Acta Clin Belg 62: 97-101, 2007

4. Bürrig KF: The endothelium of advanced arteriosclerotic plaques in humans. Arterioscler Thromb 11: 1678-1689, 1991.

5. Minamino T, Miyauchi H, Yoshida T, Ishida Y, Yoshida H and Komuro I: Endothelial cell senescence in human atherosclerosis: Role of telomere in endothelial dysfunction. Circulation 105 : 1541-1544, 2002

6. Funakoshi S, Miki K, Takaki T, Okubo C, Hatani T, Chonabayashi K, Nishikawa M, Takei I, Oishi A, Narita M, et al: Enhanced engraftment, proliferation, and therapeutic potential in heart using optimized human iPSC-derived cardiomyocytes. Sci Rep 6: 19111, 2016.

7. Neel S and Singla DK: Induced pluripotent stem (iPS) cells inhibit apoptosis and fibrosis in streptozotocin-induced diabetic rats. Mol Pharm 8: 2350-2357, 2011.

8. Yan B and Singla DK: Transplanted induced pluripotent stem cells mitigate oxidative stress and improve cardiac function through the Akt cell survival pathway in diabetic cardiomyopathy. Mol Pharm 10: 3425-3432, 2013.

9. Blin G, Nury D, Stefanovic S, Neri T, Guillevic O, Brinon B, Bellamy V, Rücker-Martin C, Barbry P, Bel A, et al: A purified population of multipotent cardiovascular progenitors derived from primate pluripotent stem cells engrafts in postmyocardial infarcted nonhuman primates. J Clin Invest 120: $1125-1139,2010$.

10. Caspi O, Huber I, Kehat I, Habib M, Arbel G, Gepstein A, Yankelson L, Aronson D, Beyar R and Gepstein L: Transplantation of human embryonic stem cell-derived cardiomyocytes improves myocardial performance in infarcted rat hearts. J Am Coll Cardiol 50: 1884-1893, 2007.

11. Khan M, Nickoloff E, Abramova T, Johnson J, Verma SK, Krishnamurthy P, Mackie AR, Vaughan E, Garikipati VN, Benedict C, et al: Embryonic stem cell-derived exosomes promote endogenous repair mechanisms and enhance cardiac function following myocardial infarction. Circ Res 117: 52-64, 2015.

12. Johnstone RM, Adam M, Hammond JR, Orr L and Turbide C: Vesicle formation during reticulocyte maturation. Association of plasma membrane activities with released vesicles (exosomes). J Biol Chem 262: 9412-9420, 1987.

13. Raposo G, Nijman HW, Stoorvogel W, Liejendekker R, Harding CV, Melief CJ and Geuze HJ: B lymphocytes secrete antigen-presenting vesicles. J Exp Med 183: 1161-1172, 1996.

14. Valadi H, Ekström K, Bossios A, Sjöstrand M, Lee JJ and Lötvall JO: Exosome-mediated transfer of mRNAs and microRNAs is a novel mechanism of genetic exchange between cells. Nat Cell Biol 9: 654-659, 2007.
15. Pascucci L, Alessandri G, Dall'Aglio C, Mercati F, Coliolo P, Bazzucchi C, Dante S, Petrini S, Curina G and Ceccarelli P: Membrane vesicles mediate pro-angiogenic activity of equine adipose-derived mesenchymal stromal cells. Vet J 202: 361-366, 2014.

16. Jeong D, Jo W, Yoon J, Kim J, Gianchandani S, Gho YS and Park J: Nanovesicles engineered from ES cells for enhanced cell proliferation. Biomaterials 35: 9302-9310, 2014.

17. Sun N, Panetta NJ, Gupta DM, Wilson KD, Lee A, Jia F, Hu S, Cherry AM, Robbins RC, Longaker MT and Wu JC: Feeder-free derivation of induced pluripotent stem cells from adult human adipose stem cells. Proc Natl Acad Sci USA 106: 15720-15725, 2009.

18. Jiang L, Yin M, Wei X, Liu J, Wang X, Niu C, Kang X, Xu J, Zhou Z, Sun S, et al: Bach1 represses Wnt/ $\beta$-catenin signaling and angiogenesis. Circ Res 117: 364-375, 2015.

19. Mallegol J, Van Niel G, Lebreton C, Lepelletier Y, Candalh C, Dugave C, Heath JK, Raposo G, Cerf-Bensussan N and Heyman M: T84-intestinal epithelial exosomes bear MHC class II/peptide complexes potentiating antigen presentation by dendritic cells Gastroenterology 132: 1866-1876, 2007.

20. Tang N, Sun B, Gupta A, Rempel H and Pulliam L: Monocyte exosomes induce adhesion molecules and cytokines via activation of NF- $\kappa$ B in endothelial cells. FASEB J 30: 3097-3106, 2016.

21. Barrès C, Blanc L, Bette-Bobillo P, André S, Mamoun R, Gabius HJ and Vidal M: Galectin-5 is bound onto the surface of rat reticulocyte exosomes and modulates vesicle uptake by macrophages. Blood 115: 696-705, 2010.

22. Nolte-'t Hoen EN, Buschow SI, Anderton SM, Stoorvogel W and Wauben MH: Activated T cells recruit exosomes secreted by dendritic cells via LFA-1. Blood 113: 1977-1981, 2009.

23. Yang G, Shi W, Hu X, Zhang J, Gong Z, Guo X, Ren Z and Zeng F: Therapeutic effects of induced pluripotent stem cells in chimeric mice with $\beta$-thalassemia. Haematologica 99: 1304-1311, 2014.

24. Wendel JS, Ye L, Tao R,Zhang J,Zhang J,Kamp TJ and Tranquillo RT: Functional effects of a tissue-engineered cardiac patch from human induced pluripotent stem cell-derived cardiomyocytes in a rat infarct model. Stem Cells Transl Med 4: 1324-1332, 2015.

25. Bang C, Batkai S, Dangwal S, Gupta SK, Foinquinos A, Holzmann A, Just A, Remke J,Zimmer K, Zeug A, et al: Cardiac fibroblast-derived microRNA passenger strand-enriched exosomes mediate cardiomyocyte hypertrophy. J Clin Invest 124: 2136-2146, 2014.

26. Xin H, Li Y, Cui Y, Yang JJ, Zhang ZG and Chopp M: Systemic administration of exosomes released from mesenchymal stromal cells promote functional recovery and neurovascular plasticity after stroke in rats. J Cereb Blood Flow Metab 33: 1711-1715, 2013.

27. Li J, Liu K, Liu Y, Xu Y, Zhang F, Yang H, Liu J, Pan T, Chen J, $\mathrm{Wu} \mathrm{M}$, et al: Exosomes mediate the cell-to-cell transmission of IFN- $\alpha$-induced antiviral activity. Nat Immunol 14: 793-803, 2013.

28. Zhang L, Zhang S, Yao J, Lowery FJ, Zhang Q, Huang WC, Li P, Li M, Wang X, Zhang C, et al: Microenvironment-induced PTEN loss by exosomal microRNA primes brain metastasis outgrowth. Nature 527: 100-104, 2015.

29. Hoshino A, Costa-Silva B, Shen TL, Rodrigues G, Hashimoto A, Tesic Mark M, Molina H, Kohsaka S, Di Giannatale A, Ceder S, et al: Tumour exosome integrins determine organotropic metastasis. Nature 527: 329-335, 2015.

30. Kiritoshi S, Nishikawa T, Sonoda K, Kukidome D, Senokuchi T, Matsuo T, Matsumura T, Tokunaga H, Brownlee M and Araki E: Reactive oxygen species from mitochondria induce cyclooxygenase- 2 gene expression in human mesangial cells: Potential role in diabetic nephropathy. Diabetes 52: 2570-2577, 2003.

31. Yu T, Robotham JL and Yoon Y: Increased production of reactive oxygen species in hyperglycemic conditions requires dynamic change of mitochondrial morphology. Proc Natl Acad Sci USA 103: 2653-2658, 2006.

32. Conigliaro A, Costa V, Lo Dico A, Saieva L, Buccheri S, Dieli F, Manno M, Raccosta S, Mancone C, Tripodi M, et al: CD90+ liver cancer cells modulate endothelial cell phenotype through the release of exosomes containing H19 lncRNA. Mol Cancer 14: 155, 2015.

33. Zhang Y, Chopp M, Meng Y, Katakowski M, Xin H, Mahmood A and Xiong Y: Effect of exosomes derived from multipluripotent mesenchymal stromal cells on functional recovery and neurovascular plasticity in rats after traumatic brain injury. J Neurosurg 122 : 856-867, 2015 .

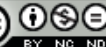

This work is licensed under a Creative Commons Attribution-NonCommercial-NoDerivatives 4.0 International (CC BY-NC-ND 4.0) License. 